\section{$\underset{\substack{\text { hommes } \\ \text { \& migrations }}}{ }$}

\section{Hommes \& migrations}

Revue française de référence sur les dynamiques

migratoires

$1304 \mid 2013$

Frontières

\title{
Territoires, souverainetés et frontières
}

Quand les dynamiques réticulaires défient les logiques étatiques

\section{Abdoulaye Diagana}

\section{(2) OpenEdition}

1 Journals

\section{Édition électronique}

URL : http://journals.openedition.org/hommesmigrations/2653

DOI : 10.4000/hommesmigrations.2653

ISSN : 2262-3353

Éditeur

Musée national de l'histoire de l'immigration

\section{Édition imprimée}

Date de publication : 1 octobre 2013

Pagination : 103-109

ISBN : 978-2-919040-24-7

ISSN : 1142-852X

Référence électronique

Abdoulaye Diagana, «Territoires, souverainetés et frontières », Hommes \& migrations [En ligne], 1304 | 2013, mis en ligne le 01 janvier 2017, consulté le 23 avril 2019. URL : http://

journals.openedition.org/hommesmigrations/2653; DOI : 10.4000/hommesmigrations.2653 
Chargement du véhicule à Kayes (Mali)

amenant les voyageurs dont Anzoumane Sissoko

au village de Monéa, Mali, 2006

@ Anaïs Pachabézian-Babel Photo 


\title{
TERRITOIRES, SOUVERAINETÉS ET FRONTIËRES QUAND LES DYNAMIQUES RÉTICULAIRES DÉFIENT LES LOGIQUES ÉTATIQUES
}

par ABDOULAYE DIAGANA, doctorant en géographie, université de Rouen, directeur du site www.kassataya.com.

\begin{abstract}
Au croisement des territoires malien, mauritanien
et sénégalais, se déploie "le pays soninké", important bassin d'émigration. Face à la nécessité de s'adapter à l'excentrement et à la "défaillance" d'États indigents, les trois régions frontalières (Kayes, Guidimakha et Tambacounda) tentent de faire de la limite frontalière un continuum fonctionnel au rythme des débits du fleuve Sénégal qui les innerve. Les populations anticipent ainsi une intégration régionale à la réalisation de laquelle elles n'ont pas été directement associées.
\end{abstract}

\section{Le contexte géopolitique}

Situés dans une bande semi-aride aux confins du Sahara, le Mali, la Mauritanie et le Sénégal font face à des conditions climatiques indigentes qui mettent l'hypothèque sur des activités économiques généralement tournées vers l'agriculture et l'élevage. La grande précarisation des conditions de vie qui a suivi la sécheresse des années 1970 a accentué la propension de certaines populations à fixer leurs regards sur des rivages réputés plus cléments. On se retrouve dès lors avec des sociétés devant faire face à des conditions de vie éprouvantes en même temps que les forces les plus productives s'en vont chercher une hypothétique fortune dans un ailleurs de plus en plus lointain. Aussi, les partenaires au développement vont-ils intégrer dans leurs politiques de coopération des volets ayant pour objectif de freiner l'hémorragie (ou l'appel d'air) en fixant les populations sur leurs territoires d'origine. La lutte contre l'immigration clandestine ${ }^{1}$ rejoint celle contre l'insécurité avec la multiplication des attaques terroristes et des prises d'otages dans le no man's land qui sépare le Mali, la Mauritanie et l'Algérie, devenu une zone de non-droit qu'écument les groupuscules liés au trafic de drogue, de cigarettes, de carburant et d'otages sous le couvert du combat religieux. Ce tournant sécuritaire et ouvertement 
intéressé fera croire un moment à l'avènement de l'ère de rapports matures et décomplexés entre des bassins d'émigration localisés en Afrique et les pays d'accueils occidentaux ${ }^{2}$, principalement au détour du discours vantant les mérites du codéveloppement à la fin de la décennie 1990. Comment, dès lors, penser le développement aux marges par la décentralisation?

\section{Les stratégies étatiques}

Les réponses apportées par les trois États à cette interrogation sont en elles-mêmes un véritable miroir de la nature des appareils étatiques en même temps qu'un baromètre de la menace centrifuge, réelle ou supposée, assumée ou refoulée. Entre le modèle malien ouvert et permissif à l'envi et le modèle mauritanien normatif à l'extrême ou le sénégalais hésitant entre
Les réponses apportées par les trois États à cette

interrogation sont en

elles-mêmes un véritable

miroir de la nature

des appareils étatiques en même temps qu'un

baromètre de la menace centrifuge, réelle ou supposée,

assumée ou refoulée. les deux, l'appareil d'État n'a ni la même prégnance ni la même évocation aux yeux des citoyens. Introduites sans concertation aucune et par la seule volonté des cabinets ministériels (au mieux) et implémentées dans le même esprit, les politiques de décentralisation au Sénégal et en Mauritanie restent hésitantes. La lecture des textes réglementant la décentralisation dans ces deux pays ne permet pas de dégager une volonté politique clairement exprimée d'un "plus de pouvoir au local", malgré la relative importance des compétences cédées sur le papier ${ }^{3}$. Le Mali présente une configuration novatrice qui tient surtout de la démarche. Les autorités d'aprèstransition ont associé les communes à lélaboration du schéma de décentralisation, y compris en leur cédant le pouvoir de délimiter leurs territoires et de s'administrer librement.

Par ailleurs, en Mauritanie et au Sénégal (le Mali dans une moindre mesure), de très fortes résistances se sont développées pour contrer les efforts de décentralisation. Elles s'adossent à des réseaux tribaux et maraboutiques qui puisent leur vigueur dans des croyances solidement ancrées et certainement de la hiérarchisation des systèmes de valeurs qui relègue la puissance publique au second rang chaque fois que nécessaire et possible.

Suscitant les passions les plus vives, le territoire est au cœur d'enjeux qui ont été le plus souvent les éléments ayant structuré les relations aussi bien inter qu'intra-entités. Le besoin d'en assurer le contrôle et la maîtrise a conduit à des crises encore vivaces (Israël-Palestine, Kosovo-Serbie) ou latentes, avec des menaces hypothéquant sérieusement la paix de régions entières à travers le monde ${ }^{4}$. Le Mali, la Mauritanie et le Sénégal ont expérimenté le phénomène, chacun à sa façon et à des degrés divers, avec des implications plus ou moins profondes.

Cependant, bien que spectaculaire (donc médiatique), ce type de conflits n'est pas celui qui vaut au continent africain les convulsions que nous lui connaissons depuis l'accession à l'indépendance de la plupart de ses États.

Que recouvrent les notions de territoire et de souveraineté pour des structures dont la création, encore récente, est probablement en devenir?

Cette interrogation, volontairement provocatrice ${ }^{5}$, invite à considérer l'organisation territoriale à l'intérieur même des entités politiques souveraines plus exactement au niveau infra que constitue le local - avec un regard sur les relations aux marges (frontalières). C'est en effet sur ces marges, à la fois floues et élastiques, que les populations tentent de traduire leur volonté de s'affirmer à travers un désir d'étendre leur contrôle sur certains territoires qui s'affranchissent des logiques étatiques. 
Quelle peut être la pertinence de telles revendications, quand on sait que, au même moment, les logiques de réseau et les flux en tous genres malmènent jusqu'à l'idée d'exclusivité territoriale ?

\section{Le territoire et son contrôle}

Les contextes qui ont vu se former et se développer le territoire respectivement en Europe et en Afrique rendent impertinente toute tentative d'établir un parallèle entre les deux conceptions du contrôle territorial. Partant, ils invitent à s'inter-

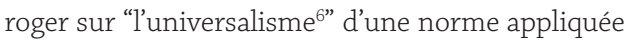
mécaniquement à des États devant accéder à une souveraineté dont les contours gagneraient à être mieux définis.

Les chemins sinueux empruntés par les nations européennes vers la formation de leurs territoires tels qu'ils se présentent aujourd'hui ont pour substrat un long mûrissement fait d'expérimentations, de conflits meurtriers et de compromis divers. Ils ont conduit de la Cité antique à l'État-nation en passant par l'Empire. Or, s'agissant de cette partie du continent africain, de nombreuses ruptures ont été opérées qui ont brutalement fait avorter les processus de construction nationale entamés bien avant l'instant colonial. Ces facteurs ont pour noms guerres de conquête et facteurs climatiques. Dans la première catégorie, figurent les campagnes d'islamisation initiées par des tribus maraboutiques $^{7}$ de l'Afrique de l'Ouest, la conquête coloniale occidentale et les campagnes négrières aussi bien des commerçants de la traite transatlantique (la mondialisation avant l'heure) que d'empereurs locaux tels l'Almamy Samory. Quant au facteur climatique, il fut déterminent dans l'affaiblissement de l'empire du Ghana ${ }^{8}$ avant les coups de boutoir des Almoravides (1071) et du Mandé (1205).

Ce que souligne Abdoulaye Bathily ${ }^{9}$ pour le Gajaaga vaut pour la plupart des anciennes entités étatiques créées avant la vulgarisation de l'écriture : il n'est pas aisé d'en délimiter avec exactitude les frontières. Celles-ci n'étaient pas représentées par des lignes soigneusement et clairement délimitées, faisant consensus et reportées sur des cartes d'état-major. La complexité des liens transversaux qui unissaient les membres des différents groupes (village, ensemble de villages, "pays", province...) était telle qu'il n'était pas possible d'établir des rapports de soumission de type pyramidal qui puissent amener à dire que telle contrée ou tel village était sous la souveraineté d'un pouvoir supérieur précis et intangible. "Un chef, qu'il fût de lignage, de village, de province ou d'État, savait bien qu'au-delà de certaines limites, son autorité ne s'exerçait plus sur les gens ${ }^{10}$."

\author{
Les chemins sinueux \\ empruntés par les nations \\ européennes vers la \\ formation de leurs territoires \\ tels qu'ils se présentent \\ aujourd'hui ont pour substrat \\ un long mûrissement fait \\ d'expérimentations, \\ de conflits meurtriers et \\ de compromis divers.
}

Lélément le plus déterminant dans la relation était sans doute plus l'emprise que pouvait avoir un souverain sur ses sujets que le fait de s'assurer le contrôle stricto sensu d'un territoire inviolable et reconnu par ses pairs comme tel. La taille dudit territoire était proportionnelle au poids démographique de l'ensemble considéré.

Par ailleurs, plusieurs facteurs rendaient difficile, voire inopérante, l'idée de frontière matérielle fixe et intangible. D'abord, la nature des liens qu'entretenaient les individus au sein du groupe. L'appartenance à la famille, au clan... prévalait sur toute autre considération. Déjà, du temps de l'empire du Ghana, les responsabilités étaient réparties en fonction du clan ${ }^{11}$. Ensuite, parce que l'activité économique (agriculture, commerce, pas- 
toralisme) était telle que les populations se déplaçaient dans des espaces géographiques qui ne connaissaient d'autres limites que celles que leur fixaient leurs besoins propres : le pasteur promenait ses troupeaux selon les saisons et l'abondance du pâturage ; le culti-
Être géographiquement

éloigné des centres de décision est sans aucun doute une circonstance qui pousse les localités à la marge à rivaliser d'ingéniosité pour développer des stratégies de sortie d'impasse. vateur se déplaçait selon ses chances d'accéder à la terre fertile, le dioula exerçait son commerce en fonction des marchés...

Il apparaît donc que les espaces politiques n'épousaient pas les frontières des espacesculturelsetmilitaires.

Comment aurait-il pu en être autrement quand onsaitqueles empiresétaienteux-mêmes mouvants

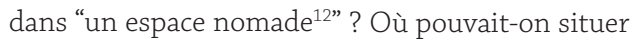
les frontières de l'empire omarien, alors même que le marabout peul avait incité ses partisans à migrer vers le Mali ? Quelles étaient les limites du village quand les cultivateurs soninké s'installaient, le temps des cultures seulement, sur le soxodebe ${ }^{13}$ qu'ils fondaient à cet effet ? Il s'agit d'un véritable casse-tête pour les cartographes : comment rendre compte de "phénomènes a priori incommensurables ${ }^{14 "}$ ?

\section{Dynamiques locales transfrontalières}

La structure urbaine des villes du Sud a fait l'objet d'une littérature abondante d'où se dégagent quelques traits généraux. Ainsi, l'étude portant sur les systèmes urbains de l'Afrique de l'Ouest intitulée "Africapolis" confirme la domination des "métropoles nationales" sur les systèmes urbains de cette partie du continent. Mais si la diffusion de l'urbanisation continue d'être surtout le fait des grandes villes, une autre métropolisation - par le bas - suscite de plus en plus d'intérêt. Les villes secondaires connaissent un dynamisme important en raison notamment de la croissance démographique favorisée par le recul de l'exode rural. Dans ce contexte, la nouvelle génération des processus de décentralisation initiés en Afrique de l'Ouest au début de la décennie 1990 offre un champ de recherche intéressant, notamment pour les régions dynamiques aux marges et souffrant de l'enclavement et de leur position excentrée.

Dans ces conditions, être géographiquement éloigné des centres de décision est sans aucun doute une circonstance qui pousse les localités à la marge à rivaliser d'ingéniosité pour développer des stratégies de sortie d'impasse. Ainsi en va-t-il de ce "pays frontière ${ }^{15}$ ", tampon entre les pays objets de cette étude et que nous circonscrivons à la région du Guidimakha en Mauritanie, au cercle de Kayes au Mali et au département de Bakel au Sénégal, en un mot une partie des anciens royaumes soninké du Gajaaga et du Guidimakha, la partie ouest de ce qu'on appelle communément "le pays soninké". Cette "aire culturelle" couvre non seulement les foyers de peuplement qui partagent l'usage de la langue soninké mais aussi les populations qui ont plus ou moins la même conception de la politique, de l'organisation sociale et du pouvoir. Nous retrouverons donc dans cette "aire" essentiellement des populations peules et maures, en plus de l'élément soninké. Replacées dans les entités étatiques modernes sous la souveraineté desquelles elles existent, les différentes localités se trouvent à plusieurs centaines de kilomètres des principaux centres de décision (les capitales Bamako, Dakar et Nouakchott). Dans un contexte où les infrastructures de transport sont

12. Denis Retaillé, “'’Espace mobile”, in Benoît Antheaume, Frédéric Giraut (dir.), Le territoire est mort. Vive les territoires! Une (re) fabrication au nom du développement, Paris, IRD, 2005, p. 82. 13. Lire " sokhodébé ". Du soninké soxo (culture) et debe (village, debu au pluriel). Le soxodebe est un site, temporaire à l'origine, installé près des terres de culture jusqu'à la saison des récoltes. Avec le temps, les soxodebu deviennent permanents en entretenant des liens suivis avec le village-jumeau (originel). 14. Ibid. 15. L'expression, qui est du président Alpha Oumar Konaré du Mali, est définie par la Direction nationale des frontières (Mali) comme "un espace géographique à cheval sur les lignes de partage de deux ou plusieurs pays limitrophes où vivent des populations liées par des rapports socio-économiques et culturels". Voir Aguibou S. Diarrah, "Le concept de "pays-frontière' dans le processus d'intégration sous-régionale ouest-africaine : résultats du séminaire de Sikasso, 4-7 mars 2002", document préparé pour la réunion du Club du Sahel et de l'Afrique de l'Ouest, tenue à Accra (Ghana), les 20 et 21 mai 2002, Dakar, Enda-Diapol, 2003. 
Anzoumane Sissoko discute avec des amis sur la route, proche de son village Monéa, Mali, 2006.

(c) Anaïs Pachabézian-Babel Photo

rares, l'enclavement de la région réduit les potentialités d'une économie tournée vers le local. Ultime interrogation : ces conditions vont-elles amener les frontières à s'estomper sous l'effet de la solidarité des excentrés?

\section{La coopération transfrontalière, une alternative à l'asphyxie des villes secondaires}

Dans la foulée des processus de décentralisation, la coopération transfrontalière s'est révélée un maillon incontournable dans la définition des politiques de développement en Afrique de l'Ouest.
Comme le souligne en janvier 2012 le rapport de l'Association des régions frontalières européennes (ARFE ou AEBR en anglais), le dispositif institutionnel en matière de coopération transfrontalière en Afrique de l'Ouest est assez fourni.

Mais des chevauchements au niveau des compétences et une insuffisance dans la coordination des politiques supra-étatiques limitent la portée desdites institutions. De plus, il y a lieu de s'interroger sur l'efficience des échelles généralement retenues pour penser les politiques de coopération transfrontalière. À l'échelle du continent, l'Union africaine (UA) - dont la Charte a opportunément gelé les frontières héritées de l'indépendance - dispose d'un arsenal d'instruments encadrant la coopération transfrontalière. La Conférence des ministres en charge des questions frontalières a réaffirmé, 
lors de sa deuxième session, à Addis-Abeba en 2010, la nécessité de "dynamiser toutes les initiatives locales de coopération transfrontalière" qu'elle se propose par ailleurs d'encourager et d'accompagner. Dans un document de synthèse intitulé "Gérer le développement en Afrique : le rôle de l'État dans la transformation économique", la United Nations

Au niveau de la sous-région ouest-africaine, la coopération

transfrontalière connaît

un certain dynamisme

institutionnel, notamment

avec la Communauté

économique des États de l'Afrique de l'Ouest (CEDEAO).
Economic Commission for Africa (UNECA) et la commission de l'UA faisaient de l'État un rouage important des politiques de développement à la condition qu'il mette l'accent sur l'intégration régionale dont le rôle est de "remédier aux difficultés liées à l'étroitesse des marchés de produits nationaux et des marchés financiers, ainsi qu'aux coûts élevés des échanges liés aux formalités de passage des frontières". L'engagement de l'UA en faveur de la coopération transfrontalière se manifeste par ailleurs à travers les communautés économiques régionales et d'autres dispositifs tel l'African Union Border Programm (AUBP). Au niveau de la sous-région ouestafricaine, la coopération transfrontalière connaît un certain dynamisme institutionnel, notamment avec la Communauté économique des États de l'Afrique de l'Ouest (CEDEAO). Le secrétariat exécutif de cette structure qui regroupe 15 États de l'Afrique de l'Ouest ${ }^{16}$ a soumis au Conseil des ministres en janvier 2005 un mémorandum intitulé "Le concept de pays frontières ou l'intégration de proximité". Ce document sert, depuis, de référence au Programme d'initiatives transfrontalières au sein de l'espace CEDEAO. Deux autres institutions opèrent dans la sous-région ouest-africaine. Il s'agit de l'Union économique et monétaire ouestafricaine (UEMOA) ${ }^{17}$ et de l'Organisation pour la mise en valeur du fleuve Sénégal (OMVS) ${ }^{18}$.

\section{Cadre institutionnel et dynamiques fonctionnelles}

Autre hypothèse, les cadres institutionnels conçus dans une approche Top Down ${ }^{19}$ n'épousent pas nécessairement les contours de l'espace fonctionnel né des pratiques quotidiennes des acteurs locaux. Les dynamiques locales aux marges ont quelque peu devancé la prospective étatique et l'échelle, trop grande, reste inadaptée au niveau micro. Pourtant, en Europe, le processus de construction d'un espace commun a également été le fait des appareils étatiques. Mais, contrairement à la région qui nous occupe, des dynamiques ont abouti à la disparition des frontières - économiques du moins - au point que les logiques d'acteurs au niveau local ne rencontrent pratiquement pas d'obstacles étatiques.

\section{Expériences d'ailleurs}

Les expériences, on le sait, ne peuvent faire l'objet de transfert en l'état. Aussi, sans être un modèle applicable prêt-à-porter, "l'Eurorégion" ${ }^{20 "}$ est-elle

16. Instituée par le traité de Lagos du 28 mai 1975, la CEDEAO est une organisation économique regroupant quinze États de l'Ouest africain : Bénin, Haute-Volta (puis Burkina-Faso), Cap-Vert (depuis 1976), Côte d'Ivoire, Gambie, Ghana, Guinée, GuinéeBissau, Liberia, Mali, Niger, Nigeria, Sénégal, Sierra Leone, Togo (pays fondateur, la Mauritanie a quitté l'organisation en 2000). La CEDEAO s'assigne pour mission principale de favoriser l'intégration économique en promouvant la constitution d'un marché interrégional. En avril 1990, elle décide de se doter d'une force d'interposition, l'ECOMOG (Economic Community of West African States Cease-fire Monitoring Group), qui compte à son actif des interventions au Liberia, en Guinée-Bissau et en Sierra Leone. 17. Héritière de l'UMOA (1962), l'Union économique et monétaire ouest-africaine est née à Dakar le 10 janvier 1994. Composée de huit États (Bénin, Burkina-Faso, Côte d'Ivoire, Guinée-Bissau depuis 1997, Mali, Niger, Sénégal, Togo), elle a pour objectif d'œuvrer à la réalisation de l'intégration des économies africaines. Elle est dotée entre autres d'une Bourse régionale des valeurs mobilières. 18. Fondée en 1971 pour exploiter les potentialités économiques du bassin-versant du fleuve Sénégal. Composée de la Guinée, du Mali, de la Mauritanie et du Sénégal, elle œuvre à rendre navigable le fleuve Sénégal et compte à son actif des ouvrages tels que les barrages de Manantali (hydroélectrique) et de Diama (anti-sel). 19. Les processus qui ont conduit à la création de ces cadres n'ont pas associé les populations concernées. Ils ont été conçus au sommet (Top) avant d'être implémentés au niveau de la base (Down). Bien que bénéficiant de l'avantage d'avoir une vision globale sur les processus, cette approche peine à emporter l'adhésion des population cibles. D'où un décalage entre les choix des élites (technocrates le plus souvent) et les attentes de la base. 20. Il s'agit d'une expérience de coopération multiforme initiée par cinq régions issues de trois pays européens. Kent, Flandre, Wallonie, Bruxelles-Capitale et Nord-Pas-de-Calais. 
une expérience de coopération transfrontalière qui poursuit des objectifs voisins de ceux des coopérations régionales africaines dans leur principe. L'expérience de la Mission opérationnelle transfrontalière (MOT) a été d'un apport déterminant en ce sens qu'elle aide les opérateurs transfrontaliers à mieux comprendre les mécanismes supranationaux et à en tirer un meilleur parti. À l'instar des régions dynamiques ouest-africaines, celles dans lesquelles agit la MOT sont "de véritables bassins de vie transfrontaliers, interdépendants" à bien des égards.

Dès lors, comment imaginer des structures de coopération qui soient adaptées aux espaces fonctionnels polarisés par les marchés frontaliers en Afrique de l'Ouest ? À l'heure de l'ouverture des frontières à l'échelle mondiale, les États de l'Afrique de l'Ouest peuvent-ils faire l'économie d'une plus grande souplesse dans la façon dont ils conçoivent l'administration des territoires frontaliers ? Les dynamiques aux marges vont dans le sens de la mise en avant des considérations économiques, politiques et sociologiques, en vue d'offrir aux territoires périphériques l'opportunité de s'aménager en fonction des synergies locales. Sous cet angle, la relation au territoire s'appréhende sous une perspective plus fonctionnelle, c'est-à-dire à l'échelle micro. Le long des régions étudiées, l'urbanisation a ouvert aux grandes villes de nouvelles opportunités qui reposent sur le dynamisme des marchés frontaliers. Mais, par la nature des activités qui y fleurissent en raison même de leur position excentrée, ces régions dynamiques souffrent de l'absence d'une coopération transfrontalière plus axée sur des problématiques qui leur sont spécifiques. La situation relance le débat sur le choix de léchelle à laquelle les activités sont encadrées en intégrant au mieux les réalités locales. 